WU B 93-15

hep-ph 9310331

Sept 1993

revised Jan 1994

\title{
Fritzsch Texture in SUSY-SO(10) with Large Neutrino Mixing
}

\author{
YOAV ACHIMAN \ and THORSTEN GREINER \& \\ Department of Physics \\ University of Wuppertal \\ Gaußstr. 20, D-42097 Wuppertal \\ Germany
}

\begin{abstract}
Fritzsch's texture is imposed on all mass matrices in a SUSY$\mathrm{SO}(10)$ via a family $U(1)_{P Q}$ symmetry. The observed charged fermion parameters fix the $\nu$-masses and mixing, while the later are evolved from the GUT scale to low energies using the RG. Large $\sin ^{2} 2 \theta_{12}$ results. As in a SUSY-GUT no intermediate scale is allowed, the RH-neutrino scale is the unification one and this gives in our model $\Delta m_{12}^{2} \approx 10^{-10} e V^{2}$, in accordance with the vacuum oscillation solution to the solar- $\nu$ puzzle.
\end{abstract}

\footnotetext{
${ }^{1}$ e-mail: achiman@wpts0.physik.uni-wuppertal.de

${ }^{2}$ e-mail: greiner@wpts0.physik.uni-wuppertal.de
} 
The general interest in supersymmetric grand unified theories (SUSY-GUTs) was revived recently in view of the observation that the gauge couplings of the standard model are unified at GUT energies when the theory is supersymmetric with $M_{S U S Y} \sim M_{Z}-1 T e V$ [任]. At the same time the new evidence for possible neutrinooscillations and the expectation that top will be observed at FERMILAB in the near future, triggered a wave of papers considering the supersymmetric extension of known and new fermionic mass models [2] [3]. Some of those papers [4] [5] deal with the $\nu$-sector also. However, SUSY does not help to solve the "Achilles heel" of the conventional GUT see-saw models: the mass matrix of the heavy RH neutrinos, $M_{\nu_{R}}$, is practically unknown in almost all those models. The conjecture of a unite matrix, diagonal matrix or a specific Ansatz for $M_{\nu_{R}}$ [6] [7] is quite arbitrary and reduces the reliability of such a theory.

The new trend in model building is to reduce the number of free parameters by using arbitrary textures. The consistent realization of such a model in terms of SUSY-GUTs, if at all possible, requires additional complicated and/or unnatural symmetries. One expects however, the mass matrices to be obtained one day from the symmetry of "the theory of everything". Hence, we think that it will be a better strategy to look for a simple symmetry which fixes the form of the mass matrices, even for the price of more parameters, especially if this tells us the form of $M_{\nu_{R}}$ as well.

One natural possibility to do this is to allow all mass matrices to have the same form. If a certain texture can then account for the observed masses and mixing it will also predict $M_{\nu_{R}}$. Now, it is well known that if an equal form for the up and down matrices is required, only one texture can account for the experimental data, namely the Fritzsch [8] one. The only problem with this was resolved recently when Babu and Shafi [3] showed that the Fritzsch texture allows for a large top mass in SUSY-GUTs. In this case, a very simple Yukawa (super-) potential suffices to give all mass matrices the same Fritzsch form and this can be induced via a special $U(1)_{P Q}$ [9] . Giving then the VEVs specific directions in the SUSY-SO(10) space, the neutrino sector will be completely given in terms of known parameters of the charged fermions.

Other special features of our model are as follows:

In contrast with most neutrino mass models, the overall scale of the RH-neutrinos, $M_{R}$, is not a free parameter in our case. We will take $M_{R}$ to be at $M_{G U T}$, in view of the fact that there is no room for an intermediate scale in SUSY-GUTs. All mass matrices, including the light neutrinos see-saw matrix, $M_{\nu}^{\text {light }}$, are then evolved using the renormalization group $(\mathrm{RG})$ from this scale to low energies, where they are confronted with the experimental data. [3

\footnotetext{
${ }^{3}$ Babu and Shafi extended recently their model for leptons also [5]. In their paper, however, the form of $M_{\nu_{R}}$ is arbitrary and fixed by hand to have a texture different from the Fritzsch one.
} 
We will give in this letter only the essential assumptions and results, the details will be published elsewhere [11].

We take the fermionic sector and the Higgs representations which contribute to the fermionic masses to be as follows:

- three families of fermions in the $\mathbf{1 6}$ representation: $\Psi_{\mathbf{1 6}}^{(i)} \quad i=1,2,3$

- a complex Higgs field in the representation 10, denoted by $H_{\mathbf{1 0}}$

- three Higgs fields in the $\mathbf{1 2 6}$ representation: $\phi_{\mathbf{1 2 6}}^{i} \quad i=1,2,3$.

We need three Higgs field combinations to obtain the Fritzsch texture:

$$
\Phi^{i}=H_{\overline{\mathbf{1 0}}}+\phi \frac{i}{\mathbf{1 2 6}}
$$

All constants in these combinations can be absorbed in the VEVs without loss of generality. The Fritzsch form is dictated by the following Yukawa (i.e superpotential) terms

$$
\mathcal{L}_{Y}=G_{12} \Psi_{\mathbf{1 6}}^{(1)} \Psi_{\mathbf{1 6}}^{(2)} \Phi^{1}+G_{23} \Psi_{\mathbf{1 6}}^{(2)} \Psi_{\mathbf{1 6}}^{(3)} \Phi^{2}+G_{33} \Psi_{\mathbf{1 6}}^{(3)} \Psi_{\mathbf{1 6}}^{(3)} \Phi^{3}+\text { h.c. }
$$

The Yukawa coupling constants will be made real by a redefinition of the relative overall phases of the fermionic representations $\Psi_{\mathbf{1 6}}^{(i)}$. Such a potential can be obtained using a specific Peccei-Quinn like $U(1)$ [12] [13.

As a result, all mass matrices will be symmetric and have the Fritzsch texture , i.e. will have the form:

$$
M_{f}=\left(\begin{array}{ccc}
0 & C_{f} & 0 \\
C_{f} & 0 & B_{f} \\
0 & B_{f} & A_{f}
\end{array}\right),
$$

where the $A_{f}, B_{f}$ and $C_{f}$ are in general complex and $f=u, d, e, \nu, \nu_{R}$. They will be fixed in terms of the VEVs of the Higgs representations.

We shall take the following VEVs directions as denoted by their $\mathrm{SU}(5)$ representation content

$$
\begin{aligned}
& <H_{10}>=r<\operatorname{along} \overline{\mathbf{5}}>+\quad p<\operatorname{along} \mathbf{5}> \\
& <\Phi_{\mathbf{1 2 6}}^{1}>=t<\operatorname{along} \overline{\mathbf{5}}>+u<\operatorname{along} \mathbf{1}> \\
& <\Phi_{\mathbf{1 2 6}}^{2}>=s<\operatorname{along} \mathbf{4 5}>+\sigma<\operatorname{along} \mathbf{1}> \\
& <\Phi_{\mathbf{1 2 6}}^{3}>=q<\operatorname{along} \overline{\mathbf{5}}>+w<\operatorname{along} \mathbf{1}>.
\end{aligned}
$$

Also, $M_{R}$ is a free parameter required to be in an intermediate scale and the light neutrino masses and mixing are not evolved. Our paper is based on the diploma thesis of one of us (T.G) [10], presented in March 1993. 
Noting now that

$<\mathbf{5}>$ gives a mass to $u$ and $\nu$ with equal weight , $\langle\overline{\mathbf{5}}\rangle$ gives a mass to $d$ and $e$ with equal weight, $<\mathbf{4 5}>$ gives a mass to $d$ and $e$ with weights $(1,-3)$,

we can explicitly write down the entries of the mass matrices.

$$
\begin{array}{lll}
A_{d}=G_{33} p & =A_{e} & \\
B_{d}=G_{23}(p+s) & \quad B_{e}=G_{23}(p-3 s) \\
C_{d}=G_{12} p & =C_{e} & \\
A_{u}=G_{33}(r+q) & A_{\nu_{D}}=G_{33}(r-3 q) \\
B_{u}=G_{23} r & =B_{\nu_{D}} & \\
C_{u}=G_{12}(r+t) & C_{\nu_{D}}=G_{12}(r-3 t)
\end{array} .
$$

Solving these equations one finds that the entries of the neutrino Dirac mass matrix $M_{\nu_{D}}$, are completely given in terms of the charged fermion mass matrices:

$$
\begin{aligned}
& A_{\nu_{D}}=-3 A_{u}+\frac{16 A_{d} B_{u}}{3 B_{d}+B_{e}} \\
& B_{\nu_{D}}=B_{u} \\
& C_{\nu_{D}}=-3 C_{u}+\frac{16 B_{u} C_{d}}{3 B_{d}+B_{e}} .
\end{aligned}
$$

The RH Majorana neutrino mass matrix, $M_{\nu_{R}}$, has also the Fritzsch texture ff in terms of

$$
A_{\nu_{R}}=G_{33} w \quad B_{\nu_{R}}=G_{23} \sigma \quad C_{\nu_{R}}=G_{12} u,
$$

where $w, \sigma$ and $u$ are the $\mathrm{SU}(5)$ singlet VEVs of $\phi_{\mathbf{1 2 6}}^{i}$ which dictate the B-L breaking in the model. They must be all of the same order of magnitude and much larger than the other VEVs. As all $\phi_{\mathbf{1 2 6}}^{i}$ come symmetrically in the Higgs potential, we

\footnotetext{
${ }^{4}$ Note the similarity of our representations and directions of the VEVs to those of Harvey, Ramond and Reiss 14. They use a different Yukawa Lagrangian, but their arguments concerning the Higgs and why the above choice of VEVs is maintained in perturbation theory, can be applicable to our case as well.

${ }^{5}$ The Fritzsch texture for $M_{\nu_{R}}$ was used already in non-SUSY models [15] in a different context with different results.
} 
shall take their VEVs to be equal in the following, for simplicity. This cannot change the general considerations.

The hierarchy in entries of the RH-neutrino mass matrix comes then from the hierarchy of the Yukawa coupling constants

$$
\begin{aligned}
& \frac{C_{\nu_{R}}}{B_{\nu_{R}}}=\frac{G_{12}}{G_{23}}=4 \frac{C_{d}}{3 B_{d}+B_{e}} \\
& \frac{B_{\nu_{R}}}{A_{\nu_{R}}}=\frac{G_{23}}{G_{33}}=\frac{1}{4} \frac{3 B_{d}+B_{e}}{A_{d}} .
\end{aligned}
$$

The $A_{\nu_{R}}$ entry dominates the RH neutrino mass matrix, $M_{\nu_{R}}$, and fixes the mass scale of this matrix. This mass scale must lie in the vicinity of $M_{G U T}$ as discussed before. Taking therefore,

$$
M_{R}=A_{\nu_{R}}=M_{G U T}
$$

there is no free parameter in the neutrino sector ${ }^{6}$.

As in the conventional SUSY-GUTs, after the GUT symmetry breaking we have effectively the minimal SUSY standard model (MSSM) between $M_{G U T}$ and $M_{S U S Y}$. In particular, two light Higgs fields survive effectively and the ratio of their effective VEVs is called, as usual, $\tan \beta$. The mass matrices of the quarks and leptons are then given by

$$
M_{u, \nu_{D}}=Y_{u, \nu_{D}} \frac{v}{\sqrt{2}} \cos \beta
$$

for up type quarks and Dirac neutrinos and

$$
M_{d, e}=Y_{d, e} \frac{v}{\sqrt{2}} \sin \beta
$$

for down type quarks and charged leptons. Here, $v=246 \mathrm{GeV}$ is the VEV of the SM-Higgs, $\tan \beta$ is a parameter and $Y_{i}$ the Yukawa matrices. We shall use in the following $A_{i}, B_{i}, C_{i}$ as elements of the Yukawa Matrices normalized according to eqs. (12) or (13), respectively.

Using a redefinition of the quark fields one can eliminate all but two phases from the quark Yukawa matrices

$$
Y_{u}=\left(\begin{array}{ccc}
0 & C_{u} & 0 \\
C_{u} & 0 & B_{u} \\
0 & B_{u} & A_{u}
\end{array}\right)
$$

\footnotetext{
${ }^{6}$ Note that, as $A_{\nu_{R}}=G_{33} \omega$ and the Yukawa coupling constant of the heavy family must be $G_{33} \simeq 1$ to get a large top quark mass, we have practically: $M_{R} \simeq \omega=u=\sigma$.
} 


$$
Y_{d}=\left(\begin{array}{ccc}
0 & C_{d} \exp (i \psi) & 0 \\
C_{d} \exp (i \psi) & 0 & B_{d} \exp (i \phi) \\
0 & B_{d} \exp (i \phi) & A_{d}
\end{array}\right)
$$

However, to obtain the best value for $\left|V_{c b}\right|$, we set as usual [3] [13], $\phi=0$. Note that $M_{d}$ and $M_{e}$ differ in the $B$ entry only. Taking however, $B_{d}=B_{e}$, as the "naive" $\mathrm{SO}(10)$ requires, does not give the right value for $m_{s}$. We found that

$$
B_{e}=-2 B_{d}
$$

results in a good fit. This relation will be used in the following discussion, with the hope to find a group theoretical explanation for it. \&

The charged lepton Yukawa matrix will be, using (15):

$$
Y_{l}=\left(\begin{array}{ccc}
0 & C_{d} & 0 \\
C_{d} & 0 & -2 B_{d} \\
0 & -2 B_{d} & A_{d}
\end{array}\right)
$$

To have the see-saw mechanism, we require that $<\Phi^{i}{ }_{\mathbf{1 2 6}}>_{\mathbf{1}}=M_{R} \approx M_{G U T}$. These will give the RH neutrinos a heavy Majorana mass.

$$
\begin{aligned}
& M_{\nu}^{\text {light }}=M_{\nu_{D}}^{T} M_{\nu_{M}}^{-1} M_{\nu_{D}} \\
& =\left(\begin{array}{ccc}
0 & \left(C_{\nu_{D}}\right)^{2} \frac{A_{d}}{C_{d}} & 0 \\
\left(C_{\nu_{D}}\right)^{2} \frac{A_{d}}{C_{d}} & \left(B_{u}-C_{\nu_{D}} \frac{B_{d}}{4 C_{d}}\right)^{2} & B_{u} A_{\nu_{D}} \frac{A_{d}}{C_{d}} \\
& \left(B_{u} C_{\nu_{D}} \frac{A_{d}}{C_{d}}\right. & \left.-A_{\nu_{D}} C_{\nu_{D}} \frac{B_{d}}{4 C_{d}}\right) \\
0 & B_{u} A_{\nu_{D}} & \left(A_{\nu_{D}}\right)^{2} \\
& \left.-A_{\nu_{D}} C_{\nu_{D}} \frac{B_{d}}{4 C_{d}}\right) &
\end{array}\right) \times M_{R}^{-1} \frac{v^{2}}{2} \sin ^{2} \beta .
\end{aligned}
$$

We are now in a position to confront this model with experiment.

The entries of the quark and charged lepton Yukawa matrices can be determined

\footnotetext{
${ }^{7}$ Note, that $\phi \simeq 0$ and $B_{e} \simeq-2 B_{d}$ will be anyhow obtained as a result of the fit in the quark sector. Hence, those statements do not effect the predictions in the neutrino sector.
} 
by fitting to the experimental values of the quark and lepton masses [17] and mixing [18]. Yet, the specific form (3) of the Yukawa matrices holds at the scale of unification $M_{G U T} \approx 10^{16} \mathrm{GeV}$. It is necessary, therefore, to use RG equations for the Yukawa couplings to evolve the matrices from $M_{G U T}$ to the experimentally accessible scale of $M_{Z}=91 \mathrm{GeV}$. We did this using two loop RG-equations for both the Yukawa and the gauge [18] couplings constants. For the Yukawa couplings the semi-analytic treatment of Barger et. al. [19 is used. The RG-equations are then solved numerically using a standard integration algorithm for differential equations. As for the see-saw neutrino matrix, $M_{\nu}^{\text {light }}$, we evolved it using one loop RG equations as in the paper of Chankowski and Ptuciennik. [20] P]

To scale both quark and lepton masses to our preferred scale of $M_{Z}$ we use three loop QCD and one loop QED RG equations.

In the Yukawa sector we used the observed masses $m_{b}, m_{c}, m_{u}, m_{e}, m_{\mu}, m_{\tau}$ and $V_{u s}$ to fix the parameters $A_{u}, B_{u}, C_{u}, A_{d}, B_{e}, C_{d}$ and $\phi$.

The experimental data used in the numerical analysis are collected in Table 1 .

Let us now summarize the results of the numerical analysis.

Keeping the SUSY breaking scale $M_{S U S Y}$ fixed, we can determine the unification scale $M_{X}$ and the unified coupling $\alpha\left(M_{X}\right)$ from the requirement that $\alpha_{1}\left(M_{X}\right)=$ $\alpha_{2}\left(M_{X}\right)$. For a SUSY breaking scale, $M_{S U S Y}=200 \mathrm{GeV}$, we found $M_{X} \approx 1.6 \times 10^{16}$ $\mathrm{GeV}$ and $\alpha\left(M_{X}\right) \approx 1 / 25$. This gives $\alpha_{3}\left(M_{Z}\right) \simeq 0.116$, in excellent agreement with the experimental data.

Only a tiny range for $\tan \beta$, is allowed for the present experimental value of $\left|V_{c b}\right|=0.030-0.058$

$$
62.7 \leq \tan \beta \leq 64
$$

The model predicts therefore the top mass as well as the mass of the s-quark.

Fig. 1 shows the dependence of $m_{t}\left(m_{t}\right)$ on $\left|V_{c b}\right|$. We find the top quark mass to be in the range

$$
94 \leq m_{t} \leq 157
$$

The strange quark mass varies only weakly with $m_{t}\left(m_{t}\right)$. We find $m_{s}(1 \mathrm{GeV})=$ $124 \pm 5 \mathrm{MeV}$ near to the lower bound of the experimental data. Note that this prediction will change once $B_{e} \neq-2 B_{d}$.

For the neutrino mixing parameters $\sin ^{2} 2 \theta_{i j}$ we find

$$
\begin{aligned}
& \sin ^{2} 2 \theta_{12}=0.991 \\
& \sin ^{2} 2 \theta_{13}=2 \times 10^{-3}-0.01 \\
& \sin ^{2} 2 \theta_{23}=0.32-1.0 .
\end{aligned}
$$

\footnotetext{
${ }^{8}$ See also 21
} 


\begin{tabular}{|c|}
\hline Gauge couplings [22 [23] \\
\hline$\alpha_{1}\left(M_{Z}\right)=0.01698 \pm 0.00009$ \\
$\alpha_{2}\left(M_{Z}\right)=0.03364 \pm 0.0002$ \\
$\alpha_{3}\left(M_{Z}\right)=0.117 \pm 0.004$ \\
\hline Quark masses [16] \\
\hline$m_{u}(1 \mathrm{GeV})=5.1 \pm 1.5 \mathrm{MeV}$ \\
$m_{d}(1 \mathrm{GeV})=8.9 \pm 2.6 \mathrm{MeV}$ \\
$m_{s}(1 \mathrm{GeV})=175 \pm 55 \mathrm{GMV}$ \\
$m_{c}\left(m_{c}\right)=1.27 \pm 0.05 \mathrm{GeV}$ \\
$m_{b}\left(m_{b}\right)=4.25 \pm 0.10 \mathrm{GeV}$ \\
\hline Lepton masses [22] \\
\hline$m_{e}(1 \mathrm{GeV})=0.4960 \mathrm{MeV}$ \\
$m_{\mu}(1 \mathrm{GeV})=104.57 \mathrm{MeV}$ \\
$m_{\tau}(1 \mathrm{GeV})=1.7835 \mathrm{GeV}$ \\
\hline $\mathrm{CKM}$ matrix entries [17] \\
\hline$V_{u s}=0.218-0.224$ \\
$V_{u b}=0.001-0.007$ \\
$V_{c b}=0.030-0.058$ \\
\hline
\end{tabular}

Table 1: The experimental data used in the numerical analysis 
The masses of the light neutrinos are as follows

$$
\begin{aligned}
& m_{1}=2.4 \times 10^{-5} \mathrm{eV}-5.3 \times 10^{-5} \mathrm{eV} \\
& m_{2}=2.6 \times 10^{-5} \mathrm{eV}-5.8 \times 10^{-5} \mathrm{eV} \\
& m_{3}=4.0 \times 10^{-3} \mathrm{eV}-1.9 \times 10^{-2} \mathrm{eV}
\end{aligned}
$$

resulting in the squared mass differences

$$
\begin{aligned}
& \Delta m_{12}^{2}=(1.0-5.9) \times 10^{-10} \mathrm{eV}^{2} \\
& \Delta m_{23}^{2}=(1.6-37) \times 10^{-5} \mathrm{eV}^{2}
\end{aligned}
$$

We see that the $\nu_{e}, \nu_{\mu}$ mixing and masses lie in the right range to solve the solar $\nu$-problem [24] [25] via vacuum oscillations [26] [27]. This solution is called sometimes "just so" 28.

We can therefore conclude as follows:

A very simple superpotential, invariant under $U(1)_{P Q}$, gives all mass matrices the Fritzsch texture and hence predicts the form of $M_{\nu_{R}}$. The overall scale of $M_{\nu_{R}}$ is taken to be $M_{G U T}$ as no intermediate scale is natural in SUSY-GUTs. The neutrino sector is then completely fixed, in our model, in terms of known parameters of the

charged fermions. All masses and mixing, including the see-saw matrix $M_{\nu}^{\text {light }}$, are evolved using the full renormalization group equations. As a result we obtain large $\nu$-mixing and $\Delta m_{12}^{2} \approx 10^{-10} \mathrm{eV}^{2}$, as is required by the "just so" solution to the solar- $\nu$ puzzle.

We thank Daniel Wicke for discussions. One of us (T.G) would like to acknowledge a graduate scholarship of the Deutsche Forschungsgemeinschaft.

\section{References}

[1] J. Ellis, S. Kelly and D.V. Nanopoulos, Phys. Lett. B 249 (1990) 441; U. Amaldi, W. de Boer and H. Fürstenau, Phys. Lett. B 260 (1991) 447; P. Langacker and M. Luo, Phys. Rev. D 44 (1991) 817.

[2] S. Dimopoulos, L.J. Hall and S. Raby, Phys. Rev. Lett. 68 (1992) 1984; Phys. Rev. D 45 (1992) 4195; H. Arason, D.J. Castaño, P. Ramond and E.J. Piard, Phys. Rev. D 47 (1993) 232; G.F. Giudice, Mod. Phys. Lett. A7 (1992) 2429; V. Barger, M.S. Berger, T. Han and M. Zralek, Phys. Rev. Lett. 68 (1992) 3394; P.Ramond, R.G. Roberts and G.G Ross, RAL-93-010.

[3] K.S. Babu and Q. Shafi, Phys. Rev. D 47 (1993) 5004. 
[4] S. Dimopoulos, L.J. Hall and S. Raby, Berkeley Preprint UCB-PTH-92-21; M. Fukugita, M. Tanimoto and T. Yanagida, Kyoto Preprint YITP/K-983.

[5] K.S. Babu and Q. Shafi, Bartol Preprint, BA-93-09

[6] For reviews see: H. Harari and Y. Nir, Nucl. Phys. B 292 (1987) 251; P. Langacker, PEN Preprint UPR-0552T.

[7] K.S. Babu and Q. Shafi Phys. Lett. B 294 (1992) 235; M. Cvetic and P. Langacker, Phys. Rev. D 46 (1992) R2759; J. Ellis, J. Lopez and D.V. Nanopoulos, Phys. Lett. B 292 (1992) 189.

[8] H. Fritzsch,Phys. Lett. B 70 (1977) 436; Phys. Lett. B 73 (1978) 317; Nucl. Phys. B 155 (1979) 189;

See also: S. Weinberg, in "A Festschrift for I.I. Rabi" [Trans. N.Y. Acad. Sci., Ser. II (1977), v. 38], p. 185; F. Wilczek and A. Zee, Phys. Lett. B 70 (1977) 418.

[9] R. Peccei and H. Quinn, Phys. Rev. Lett. 38 (1977) 1440.

[10] T. Greiner, diploma thesis Wuppertal Preprint WU B 93-15, March 1993.

[11] Y. Achiman and T. Greiner, in preparation.

[12] A. Davidson and K.C. Wali Phys. Rev. Lett. 48 (1981) 11.

[13] H. Georgi, A. Nelson and M. Shin, Phys. Rev. Lett. 150 (1984) 306; M. Shin, Phys. Lett. B 154 (1985) 205; Phys. Lett. B 160 (1985) 411.

[14] J. Harvey, P. Ramond and D. Reiss, Phys. Lett. B 92 (1980) 309; Nucl. Phys. B 199 (1982).

[15] K. Kang and M. Shin, Phys. Lett. B 185 (1987) 163.

[16] J. Gasser and H. Leutwyler, Phys. Rep. 87 C (1982) 77; H. Leutwyler, Nucl. Phys. B 337 (1990) 108; S. Narison, Phys. Lett. B 216 (1989) 191.

[17] Particle Data Group, J.J. Hernández et al., Phys. Lett. B 239 (1990) 1.

[18] M.E. Machachek, M.T. Vaughn, Nucl. Phys. B 232 (1983) 83; J.E. Björkman, D. R. T. Jones, Nucl. Phys. B 259 (1985) 533

[19] V. Barger, M.S. Berger, P. Ohmann, Madison Preprint MAD/PH/722; see also E. Ma, S. Pakvasa, Phys. Lett. B 86(1979) 43; PRD20792899; K. Sasaki, Z. Phys. C 32 (1986) 149; K. S. Babu, Z. Phys. C 35(1987) 69.

[20] P.H. Chankowski, Z. Płuciennik, Preprint ZU-TH 20/93.

[21] K.S. Babu, C.N. Leung, J. Pantaleone, Preprint IUHET-252. 
[22] H. Arason, D.J. Castaño, B. E. Keszthelyi, S. Mikaelian, E.J. Piard, P. Ramond, B. D. Wright, Phys. Rev. Lett. 67 (1991) 2933.

[23] S. Bethke, S. Catani, CERN Preprint CERN-TH.6484/92, 1992

[24] J.N.Bahcall, Neutrino Astrophysics (Cambridge Univ. Press, Cambridge, England, 1989).

[25] For a recent review see: A.Yu. Smirnov, Talk given at the XVI Symposium "Lepton-Photon Interactions" Cornell Univ. August 1993.

[26] S.M. Bilenky and B.M. Pontecorvo, Phys. Lett. B 41 (1978) 225.

[27] For a recent calculation see: P.I. Krastev and S.T. Petcov, SISSA- preprint 177/93/EP ,1993.

[28] S.L.Glashow and L.M.Krauss, Phys. Lett. B 190 (1988) 199.

\section{Figure Captions}

Fig. $1: m_{t}\left(m_{t}\right)$ dependence on $\left|V_{c b}\right|$.

Fig. 2 : The neutrino masses as a function of $m_{t}\left(m_{t}\right)$.

Fig. 3 : The dependence of the squared mass difference $\Delta m_{12}^{2}$ on the mixing parameter $\sin ^{2} 2 \theta_{12}$. 
This figure "fig1-1.png" is available in "png" format from: http://arxiv.org/ps/hep-ph/9310331v2 